\title{
Combined Electrostatic-Covalent Building of Au NPs Multilayers and Their Size-Enhanced Cohesive and SERS Properties
}

\author{
K. Mougin, S. Darwich, L. Vidal, and H. Haidara \\ Institut de Science des Matériaux de Mulhouse, IS2M-CNRS/UHA, 15 Rue Jean Starcky, 68057 Mulhouse, France \\ Correspondence should be addressed to K. Mougin, karine.mougin@uha.fr
}

Received 21 September 2010; Revised 9 November 2010; Accepted 30 December 2010

Academic Editor: Konstantin Neyman

Copyright $\odot 2010 \mathrm{~K}$. Mougin et al. This is an open access article distributed under the Creative Commons Attribution License, which permits unrestricted use, distribution, and reproduction in any medium, provided the original work is properly cited.

Multilayers of well-ordered and close-packed 2D nanostructures of gold nanoparticles (NPs) were fabricated using a layer-by-layer technique. Colloidal spherical Au NPs of 5 and $15 \mathrm{~nm}$ diameters were synthesized and, respectively, self-assembled in multilayers. The robustness of these systems was insured by a combination of electrostatic and covalent bonds between nanoparticles and linker molecules. The compacity of the superlattice was characterized by AFM observation and ellipsometry measurements. Evidence of stronger cohesion of multilayers of smaller NPs size was brought by submitting the systems to sonication test. The multilayers have also proved analytical potential when used to detect low concentration methylene blue molecules adsorbed on the Au nanoparticles, by surface-enhanced Raman spectroscopy (SERS). The detection sensitivity of these two sized Au NPs architectures was directly compared to an evaporated "bulk" Au thin film of equivalent thickness. Results have displayed a strong increase of the electromagnetic field enhancement with a decrease of the NPs size, whereas the bulk thin Au film was shown to be inefficient as a SERS substrate. These results bring a nice evidence of size effects on the global performance (SERS, cohesion) and hopefully on the stability of NPs based nanostructures.

\section{Introduction}

In recent years, considerable effort has been devoted to the design and fabrication of structured materials with functional properties. The long-range and large-scale arrangement of nanoparticles (NPs) is an important issue in the development of nanostructured materials with new electronic, magnetic, and photonic properties [1]. The two- (2D) and three-dimensional (3D) arrangements of nanoparticles still remain difficult for certain particles shapes (anisotropic rods, ellipsoidal, stars, etc.). Different top-down techniques like photolithography [2] and microcontact printing [3] have been developed to organize nanoparticles on substrates.

In this field, noble metal nanoparticles, and especially gold nanoparticles, have attracted a great deal of interest. In particular, supported nanostructured films of Au NPs have appeared as promising functional structures for applications as diverse as optical field enhancement $[4,5]$ and catalysis $[6-$ 8]. Indeed, metal particles of size smaller than the wavelength of light exhibit specific optical properties due to the resonant excitation of coherent electron oscillations, a phenomenon known as localized surface plasmons. When used to enhance the optical susceptibility of molecules that are embedded in a nanoparticulate surface which is irradiated by an electromagnetic field (light), this optical property (surface plasmons) can be used as a powerful spectroscopic tool, also known as surface-enhanced Raman spectroscopy (SERS).

However, the cooperative effect of these surface plasmons as well as the robustness of the multilayer structure will strongly depend on the interactions between the different building blocks (nanoparticle/nanoparticle, nanoparticle/linker) which determine the overall organization of the network.

For the elaboration of such nanoparticulate multilayers, there are two main approaches: a chemical one via colloids synthesis and assembly and a more conventional one based on chemical vapor deposition (CVD) procedure. One of the advantages of colloidal assembly is the way the particle size, shape, and chemistry can be easily adjusted. For instance, nanocolloidal Au particles can be prepared with diameters ranging from 1 to $120 \mathrm{~nm}$ with a relatively high monodispersity. As a result, surfaces made from colloidal $\mathrm{Au}$ 


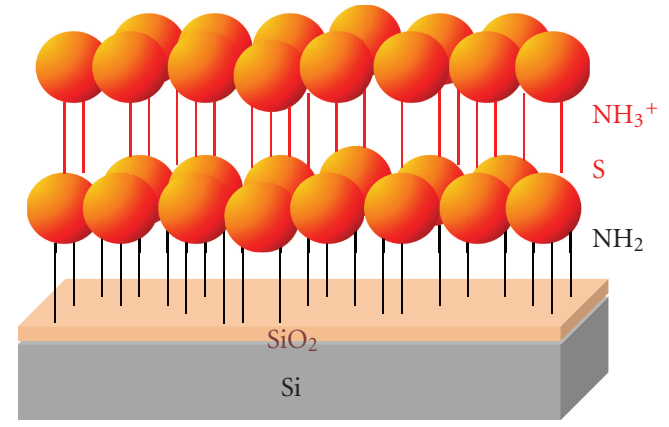

Figure 1: Schematic representation of the combined electrostaticcovalent layer-by-layer (LbL) formation of Au NPs multilayers (relative size of linker and NPs are not at scale).

NPs have a tunable nanometer-scale roughness determined solely by the particle diameter and packing. The ultimate surface and bulk properties of these nanoparticulate surfaces will be directly correlated to these intrinsic parameters of the individual NPs (nature, size, shape, functionalization).

In the present work, the nanoparticulate multilayer superlattice was prepared using the principle of alternating adsorption (layer-by-layer procedure) which was introduced in 1966 by Iler from colloidal particles of opposite charges [9]. This method known as layer-by-layer (LbL) deposition is based on electrostatic interactions between polyanions and polycations. Although Iler's work did not attract much attention at that time, it was one of the first studies that successfully demonstrated that thin films of nanoparticles could be readily assembled on rigid supports. Twenty-five years after Iler's discovery, Decher and coworkers utilized LbL technique to fabricate multilayer thin films from oppositely charged polyelectrolytes [10]. Hu and coworkers have also developed a facile approach for the preparation of multilayered nanostructure of gold nanorods using a linear polyethylenimine as a polymeric adhesive layer [11].

This LbL procedure has therefore inspired our work although we have developed here a less conventional approach based on the use of two alternating and distinct bonds between the consecutive layers to assemble the Au particles in the multilayer. Two sizes of spherical nanoparticles were used ( 5 and $15 \mathrm{~nm}$ diameter) to build two different multilayer systems. The particles were transferred from the nanocolloidal solution onto self-assembled monolayers (SAMs) coated silicon wafers, using dip-coating process. Two consecutive NPs layers were tightly bonded which was insured by the combination of two alternating and distinct interactions. Basically, an electrostatic interaction was used to bind the charge-stabilized (citrates) colloidal gold nanoparticles to the terminal amine of an alkylthiol linker. The second binding is a covalent one $(\mathrm{Au} / \mathrm{S})$ that attaches the second layer of Au particles to the terminal sulfur (S) of the alkylthiol linker, as shown in Figure 1.

Compared to previous routes of structuring NPs into supported multilayers, this approach has several distinguished advantages: (i) aqueous solutions of colloidal Au NPs are easy to prepare and are stable for long periods of time, (ii) colloidal Au NPs can be prepared with a wide range of shape and size, (iii) multilayers of nanoparticles can be assembled easily using a layer-by-layer technique, and (iv) the distance between NPs can be controlled by adjusting the length of the linker molecule.

Finally, these nanoparticulate thin films were assessed for their size-dependent cohesive and optical (SERS) properties. The robustness of both sized nanostructured multilayer systems has been tested by submitting them to mechanical vibrations provided by sonication during several tens of minutes. The optical SERS sensitivity of the multilayer systems versus the size of the constitutive particles was assessed, using blue methylene as a model analyte molecule. These results were discussed for their dependence on nanoparticle size in the supported multilayers, compared to those obtained on a uniform Au film of identical thickness, and deposited by thermal evaporation.

\section{Experimental Methods}

Gold nanoparticles were prepared using a colloidal procedure. Citrate-stabilized Au nanoparticles were synthesized according to a procedure published by Frens [12].

2.1. Preparation of Colloidal Particles. An aqueous solution $(50 \mathrm{~mL})$ of tetrachloroauric (III) acid hydrate $\mathrm{HAuCl}_{4} \cdot \mathrm{H}_{2} \mathrm{O}$ at $3 \%$ weight was prepared (solution A). This auric solution was reduced and stabilized with citric acid trisodium, by adding $1.25 \mathrm{~mL}$ of $1 \%$ weight aqueous solution of citric acid trisodium (solution $\mathrm{B}$ ) to the previous one, under strong stirring during $15 \mathrm{~min}$.

The result of this reaction is a red suspension of spherical nanogold particles of average size $15 \mathrm{~nm}$ (Figure 2(a)).

$0.75 \% \mathrm{NaBH} 4$ solution dissolved in a $1 \%$ sodium citrate solution was prepared (solution C). $1.25 \mathrm{~mL}$ of solution B was added to solution $\mathrm{A}$ under strong stirring. Then $1 \mathrm{~mL}$ of solution $\mathrm{C}$ was added consecutively and stirred for additional $5 \mathrm{~min}$. The resulting colloidal solution is a dark blue solution of nanogold particles of average size of $5 \mathrm{~nm}$ (Figure 2(b)).

It is worth mentioning here that the average particle size (mean value and dispersion) is highly sensitive to slight variations in the processing parameters, such as mixing rate, temperature, humidity, and ageing of the reagents as shown in Figures 3(a) and 3(b).

The principle of the elaboration of the NPs multilayers is based on a layer-by-layer (LbL) deposition technique that consists essentially in the following steps

The substrate, a silicon $(\mathrm{Si})$ wafer bearing its native silica $\left(\mathrm{SiO}_{2}\right)$ layer of $\sim 2 \mathrm{~nm}$, was coated with a self-assembled monolayer (SAM) of an $\mathrm{NH}_{2}$-terminated silane molecule, $n$-(6-aminohexyl)aminopropyltrimethoxysilane $\left(\mathrm{OCH}_{3}\right)_{3}$ $\mathrm{Si}\left(\mathrm{CH}_{2}\right)_{3}-\mathrm{NH}-\left(\mathrm{CH}_{2}\right)_{6}-\mathrm{NH}_{2}$. This $\mathrm{NH}_{2}$-terminated SAM surface is here referred to as $\mathrm{NH}_{2}$. A standard chemical procedure of self-assembling of organosilanes onto hydroxylated substrate was used for this purpose. The silicon wafer was first cleaned in $3: 7(\mathrm{v} / \mathrm{v})$ mixture of $30 \%$ $\mathrm{H}_{2} \mathrm{O}_{2} / \mathrm{H}_{2} \mathrm{SO}_{4}$ and thermostated at $50^{\circ} \mathrm{C}$ for $30 \mathrm{~min}$ (caution: these solutions react violently with organic materials and 


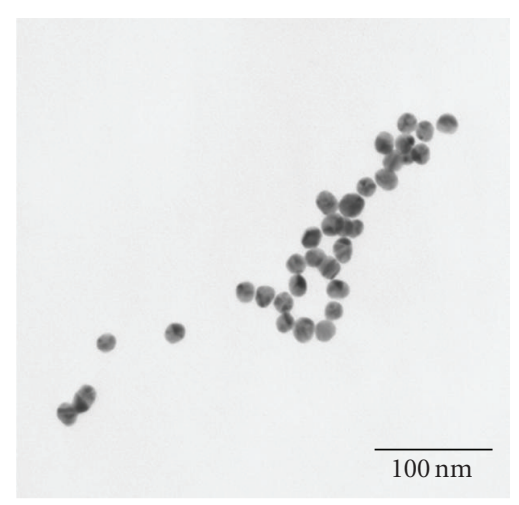

(a)

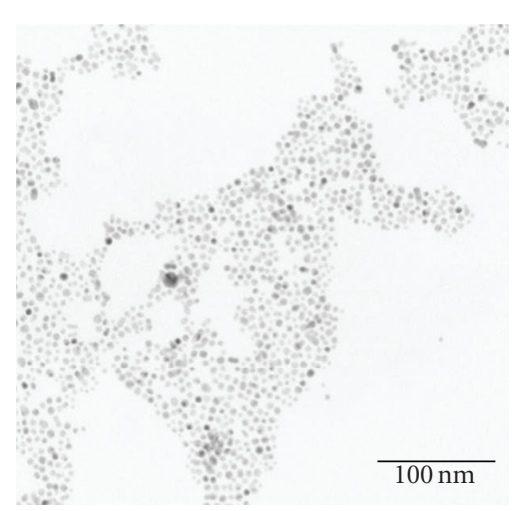

(b)

FIgURE 2: TEM images of a homogeneous size distribution of spherical Au nanoparticles (scale bar 100 nm): (a) average diameter of $15 \pm$ $3 \mathrm{~nm}$, (b) average diameter of $5 \pm 1 \mathrm{~nm}$.

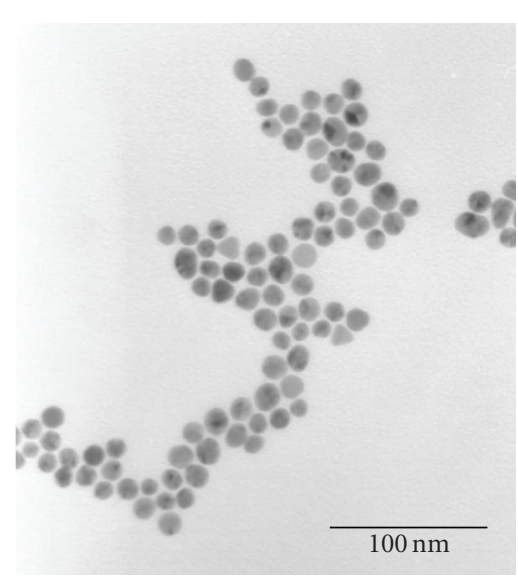

(a)

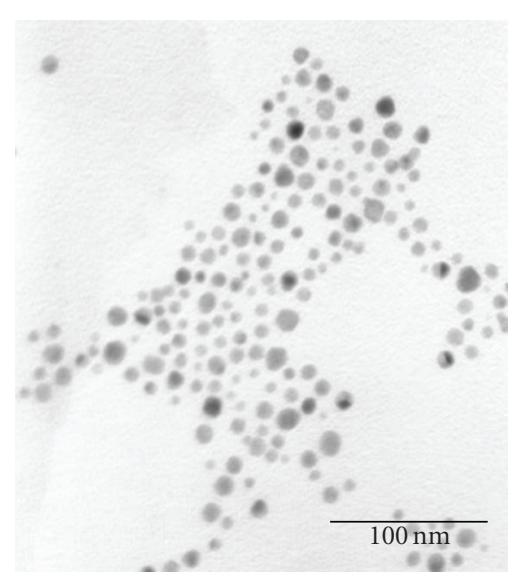

(b)

FIgURE 3: TEM images of Au nanoparticles of an average diameter of $15 \mathrm{~nm}$ (a) with a homogeneous size distribution (scale bar $100 \mathrm{~nm}$ ), (b) with a heterogeneous size distribution (scale bar $100 \mathrm{~nm}$ ) due to the use of a lower operating temperature $\left(60^{\circ} \mathrm{C}\right.$ instead of $\left.80^{\circ} \mathrm{C}\right)$ in the synthesis process.

must be handled with extreme caution!!!). The wafers were thoroughly rinsed, sonicated several times in Milli-Q water, and dried under nitrogen flow. This treatment produced a surface with a high coverage of silanol $(\mathrm{SiOH})$, to which functional silanes could adsorb and self-assemble upon hydrolysis and condensation. The Si wafers were then soaked at least 6 hours in a $5 \mathrm{mM}$ ethanol solution of $n$-(6-aminohexyl)-aminopropyltrimethoxysilane. After the completion of the coating, the samples are rinsed and sonicated for about $5 \mathrm{sec}$ with the same solvent to remove weakly attached molecules and then dried under a nitrogen flow.

2.2. Formation of the First Layer of Gold Nanoparticles. The sample was immersed in the solution of Au nanoparticles of either $15 \mathrm{~nm}$ or $5 \mathrm{~nm}$ diameter for 15 to 20 minutes (optimal time to reach a compact layer of nanoparticles). Then, it was rinsed and sonicated in water for about 1 minute in an ultrasonic bath. Finally, the sample was rinsed again with water and dried under nitrogen flow.

\subsection{Desorption of the Stabilizing Citrate Molecules Adsorbed} on the Au Particles. This first step is aimed at cleaning the Au NPs to obtain a bare gold surface. The organic citrate molecules adsorbed on the Au NPs (Figure 4) were etched by irradiating the first Au layer bearing these molecules with ultraviolet light (UV) " 254 nm” over night. The resulting sample of bare and clean Au NPs was then immersed in $1 \mathrm{mM}$ solution of "11-amino-1-undecanehiol, hydrochloride" $\left(\mathrm{C}_{11} \mathrm{H}_{25} \mathrm{NS} \cdot \mathrm{HCl}\right)$ in chloroform, for 3 hours. This step leads to the formation of thiolate bonds ( $\mathrm{S}$ $\mathrm{Au}$ ) between the Au NPs and the linker molecule bearing at the other end $\mathrm{NH}_{2}$ terminal groups. This operation finally produces a functional surface similar to the initial $\mathrm{NH}_{2}$-terminated self-assembled monolayer, coating the $\mathrm{Si}$ wafer. 


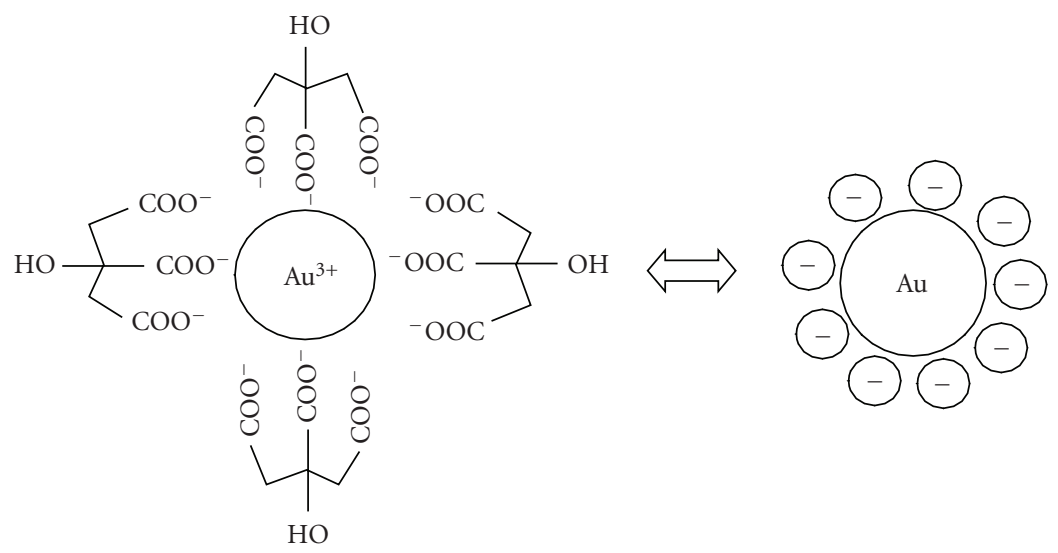

FIGURE 4: Schematic representation of the charge-stabilized (citrates) colloidal gold nanoparticles (relative size of stabilizing molecules and nanoparticles are not at scale).

2.4. Formation of the Second Layer of Gold Nanoparticles. The above $\mathrm{Au}$ NPs layer bearing $\mathrm{NH}_{2}$ terminal groups is re-immersed in the same solution of colloidal nanoparticles for 15 to 20 minutes, producing, as in the first step, a second citrate-stabilized Au layer, via $\mathrm{NH}_{2}$ /citrate (negatively charged $\mathrm{COO}^{-}$) bonds. The following steps for the multilayer construction consist in repeating the above alternate cycle.

\section{Results and Discussion}

Gold colloidal nanoparticles were first characterized by transmission electron microscopy (TEM) and UV-Vis spectrophotometry in order to determine the morphology and size of the gold nanoparticles. Secondly, the Au NPs multilayers were characterized by AFM and ellipsometry to confirm the formation of the layered structures, their compactness, and the spatial organization of the NPs.

\subsection{Characterization of Gold Nanoparticles}

3.1.1. TEM Characterization. As shown in Figure 2, transmission electron microscopy (TEM) observations well confirmed the spherical shape of the Au NPs, as expected from the used synthesis procedure. From the analysis of the TEM images, the average diameter of the nanoparticles in the two solutions was found to be $15 \pm 3 \mathrm{~nm}$ and $5 \mathrm{~nm} \pm 1 \mathrm{~nm}$.

3.1.2. UV-Vis Spectrophotometry of Colloidal Solution. The $15 \mathrm{~nm}$ colloidal dispersions show an absorption band at about $519 \mathrm{~nm}$ in the UV-visible spectrum (Figure 5). This band arises from the excitation of the conduction electrons, localized at the surface of isolated particles, and usually known as "surface plasmon resonance" [13]. The $5 \mathrm{~nm}$ diameter solutions display a band with a maximum absorption peak at $497 \mathrm{~nm}$. This shift in the absorption band with the particle size is in agreement with literature for spherical gold nanoparticles [14]. However, Au nanocolloids can also undergo aggregation by aging or by ligand linkage, leading

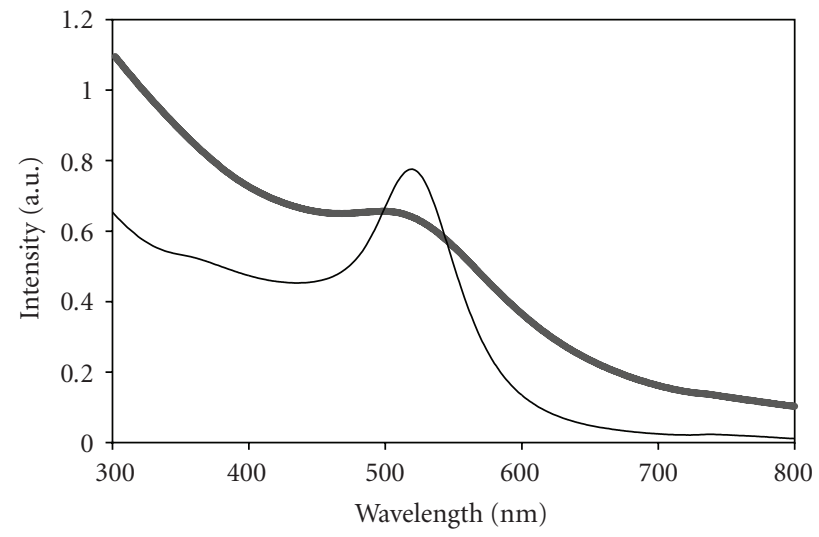

Figure 5: UV-Vis absorption spectra of gold colloidal solutions. The thin line corresponds to the spectral response of the nanocolloidal solution of average particles size of $15 \mathrm{~nm}$, as previously determined by TEM in Figure 3. The broad line represents the nanocolloidal solution of average particles size of $5 \mathrm{~nm}$, as previously determined by TEM in Figure 3.

equally to a shift of the absorption peak towards lower energies (higher absorption wavelengths).

3.2. Characterization of Gold Particulate Layers. The gold nanoparticles were deposited into multilayers architecture and characterized by ellipsometry and AFM to verify, respectively, the homogeneity and morphology of the NPs assembly.

3.2.1. Ellipsometry. For both multilayer systems made respectively, of 15 and $5 \mathrm{~nm}$ diameter particles, the thickness of each NPs layer has been measured, using a Null-Ellipsometer (Multiskop, Optrel, Germany), working at $532 \mathrm{~nm}$ (Nd-YAG laser), in the "laser-polarizercompensator-sample-analyzer" arrangement. For the ellipsometric modeling, each layer is represented by a monolayer of Au nanoparticles with its tethered thiolate molecular film (linker). The thickness $(t)$ of the nanoparticulate film at 
TABLE 1: Evaluation of the complex refractive index $(n, k)$ of NPs multilayer structure and a thermally evaporated Au film by ellipsometry.

\begin{tabular}{lcc}
\hline & Refractive index $n$ & Extinction coefficient $k$ \\
\hline Au evaporated film $30 \mathrm{~nm}$ & 0.488 & -2.4 \\
$5 \mathrm{~nm}$ Au colloidal 1 layer equivalent to $5 \mathrm{~nm}$ & 3.814 & -0.896 \\
$5 \mathrm{~nm}$ Au colloidal 4 layers equivalent to $20 \mathrm{~nm}$ & 3.014 & -1.664 \\
$15 \mathrm{~nm}$ Au colloidal 4 layers equivalent to $60 \mathrm{~nm}$ & 3.045 & -1.522 \\
\hline
\end{tabular}

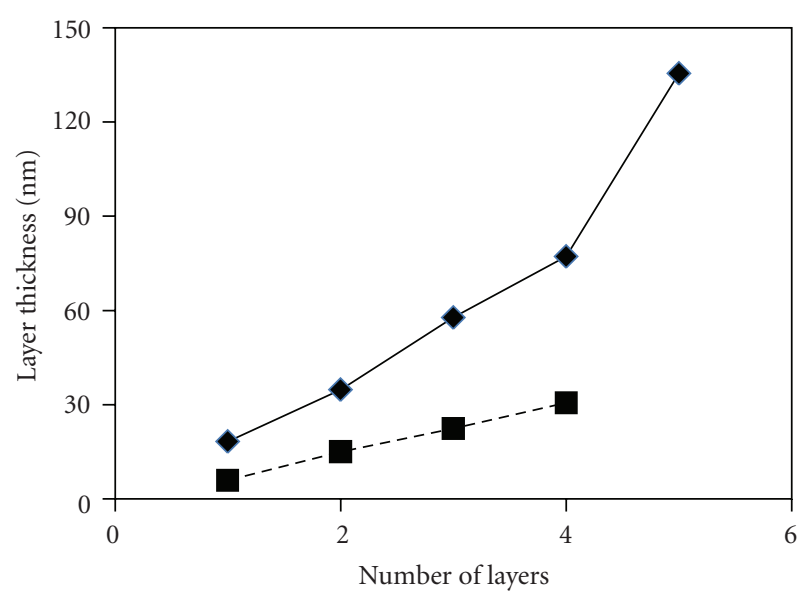

Figure 6: Evolution of the multilayer film thickness versus the number of layers. The measurements were done by ellipsometry. One layer is made of a monolayer of gold nanoparticles with its tethered thiolate molecular film (linker). Dotted line $(5 \mathrm{~nm}$ size $\mathrm{NPs}$ ), continuous line (15 $\mathrm{nm}$ size NPs).

the different layering steps and the corresponding complex refractive index $(n, k)$ were determined from the measured ellipsometric angles, using the resident Elli software.

The thickness $(t)$ and refractive index $(n, k)$ of the first layer were first determined. And for each additional layer, the refractive index of the new multilayer system was recalculated. It should also be mentioned here that the convergence of the layer model defined in the Elli software reaches its limit over three interfaces. In addition, the measurements of the refractive index of the Au NPs multilayers show that they do not display a bulk feature $\left(n=0.43 ; k=-2.455\right.$ for a $\left.\lambda_{\text {laser }}=532 \mathrm{~nm}\right)$. For the $5 \mathrm{~nm}$ nanoparticles, the average refractive index for the first layer was $n=3.5 ; k=-1.0$ as displayed in Table 1. For the first layer of the $15 \mathrm{~nm}$ and $5 \mathrm{~nm} r$ Au NPs, we obtained an average thickness of, respectively, $19.0 \pm 0.5 \mathrm{~nm}$ and 6.0 $\pm 0.5 \mathrm{~nm}$, using an approximate value of the film refractive index of 3 and an extinction coefficient of -1 (Figure 6). Those results of optical constants are very different from the Au bulk parameters reported in the literature [15].

However, it is well known that dielectric constants are independent of film thickness above a critical thickness, which for gold is around $25 \mathrm{~nm}[12,16]$. The nanoparticulate film presents voids in between nanoparticles which enables transmission of the light up to the silicon substrate. This sensitivity of the substrate has a strong impact on the value of the global optical constants. The refractive index of the Si substrate $(n=4.23 ; k=-0.043$ for at $532 \mathrm{~nm})$, which seems to be accessible to the laser light, creates a composite index of the multilayer. The combined effect of the voids $\left(n_{\text {air }}=1\right)$ and of Si substrate underneath results in a much higher $n_{\text {effective }}$ and a much lower absorbance $\left|k_{\text {effective }}\right|$ for the Au NPs multilayers, compared to bulk Au film.

However, we note that our ellipsometry film thicknesses are in average larger by $\sim 1-2 \mathrm{~nm}$ than estimated from the diameter of the NPs. This may arise from the presence of a few aggregates averaging the outmost layer.

To compare the effectiveness of our multilayer structure to a standard bulk thin gold film, we have prepared $\mathrm{Au}$ thin films by thermal evaporation. Contrary to previous results, the optical constants of $\mathrm{Au}$ evaporated thin films are close to those expected for bulk Au material, due to the higher compactness of slowly evaporated thin films.

3.2.2. AFM in Tapping Mode. Tapping mode AFM in ambient conditions has been used to characterize both the particle size and the morphology of the multilayers (packing, spatial organization). Figure 7 presents the organization of nanoparticles, layer after layer on the same sample (step-bystep adsorption) for bothparticle sizes, 15 and $5 \mathrm{~nm}$.

For the first layer of Au NPs (15 or $5 \mathrm{~nm}$ ), AFM observations show well-ordered and close-packed surface structures as it appears respectively, in Figures $7(a)$ and $7(d)$. Figures $7(\mathrm{~b}), 7(\mathrm{c}), 7(\mathrm{e})$, and $7(\mathrm{f})$ show the $2 \mathrm{D}$ organization of the additional layers. The average roughness for the two different multilayer structures is $5.0 \pm 0.5 \mathrm{~nm}$ and $1.0 \pm$ $0.5 \mathrm{~nm}$, respectively, for the 15 and $5 \mathrm{~nm}$ NPs. These values indicate that the layered NPs are compact and uniform with very few aggregates, insuring a high surface coverage. This dense organization of the nanoparticles can be explained by the combination of the two alternating strong bonds that link the consecutive NPs layers: the covalent bond between the Au NPs and the end-sulfur (S) atom of the alkylthiol linker, and the electrostatic one between the $\mathrm{NH}_{2}$ head group of the linker and the carboxylate $\left(\mathrm{COO}^{-}\right)$from the charge stabilizing citrate of the Au NPs. Finally, both the measured ellipsometric film thickness and the AFM image seem to consistently support the close packing of the multilayer structures.

These functional multilayered structures have been tested for their cohesion and adhesion using mechanical vibrations provided by a sonicator bath (Fisherbrand Ultrasonic cleaner, $35 \mathrm{kHz}, 40 \mathrm{~W}$ ) for several tens of minutes. AFM images have been carried out before and after the mechanical test. The results (displayed in Figure 8) show that the $15 \mathrm{~nm}$ size multilayer system starts to delaminate after 20 minutes 


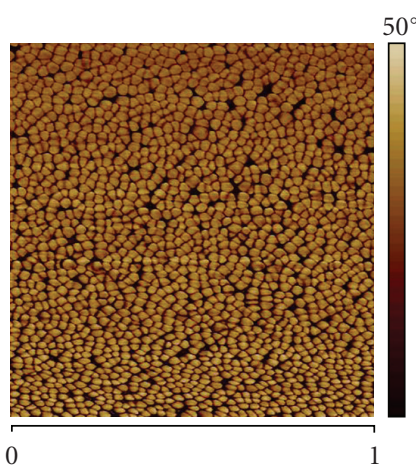

Phase $(\mu \mathrm{m})$

(a)

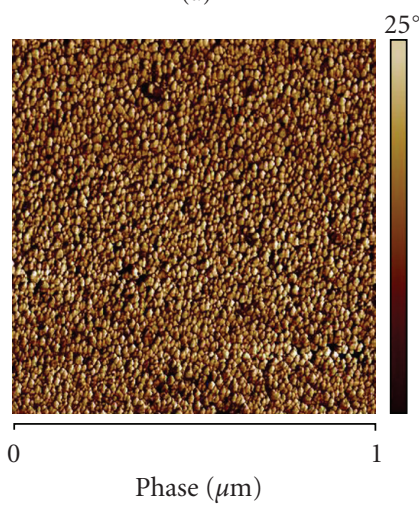

(d)

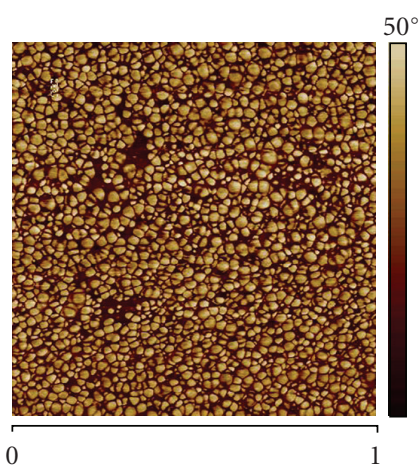

Phase $(\mu \mathrm{m})$

(b)

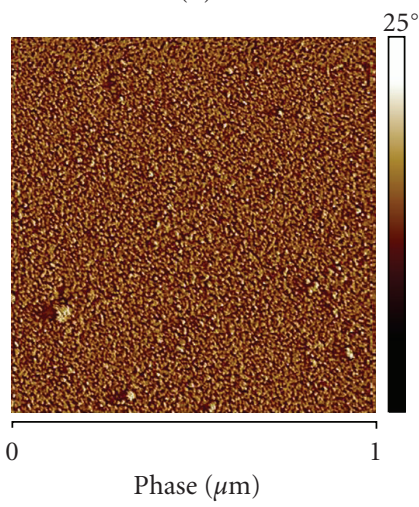

(e)

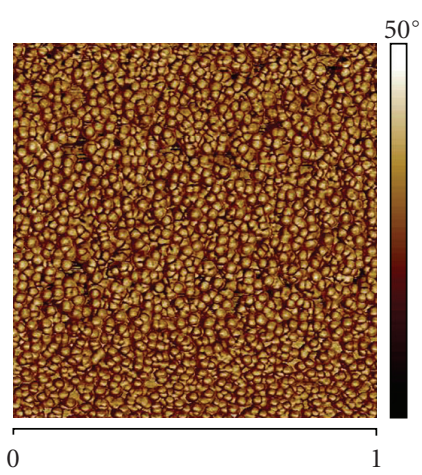

Phase $(\mu \mathrm{m})$

(c)

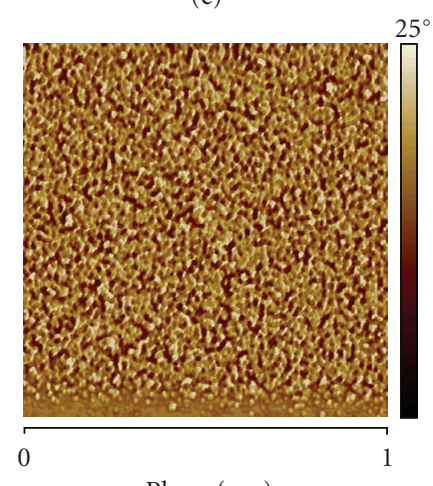

Phase $(\mu \mathrm{m})$

(f)
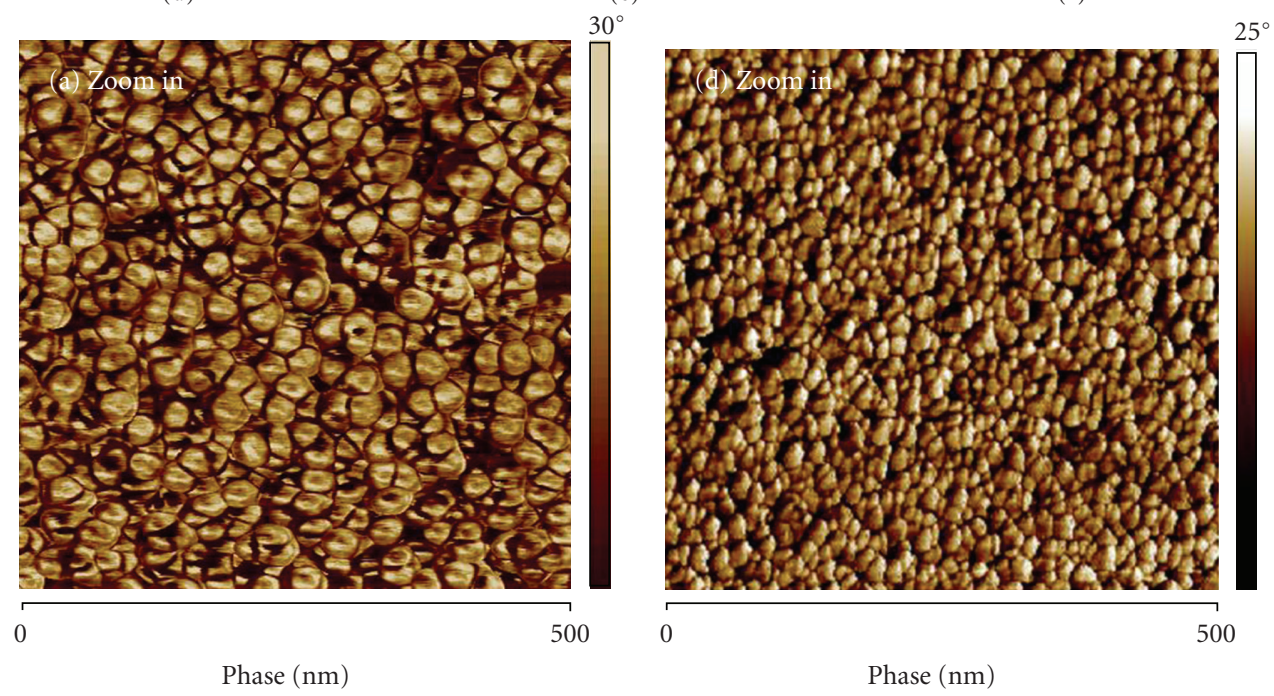

FIGURE 7: AFM pictures in tapping mode of the last NPs layer at the different (successive) steps of the formation of the complete multilayer structure on the substrate. From (a) to (c), the images correspond to the $15 \mathrm{~nm}$-sized NPs, and from (d) to (f), to the $5 \mathrm{~nm}$ NPs. $15 \mathrm{~nm}$ NPs: (a) first layer, zoom in: $500 \times 500 \mathrm{~nm}^{2}$ AFM phase contrast image of a zoom in of the 2D-assembly of the NPs, (b) second layer, (c) fourth layer; the scale is $(1 \times 1 \mu \mathrm{m}) ; 5 \mathrm{~nm}$ NPs: (d) first layer, zoom in: $500 \times 500 \mathrm{~nm}^{2}$ AFM phase contrast image of a zoom in of the 2D-assembly of the NPs. (e) Second layer, (f) fourth layer; the scale is $(1 \times 1 \mu \mathrm{m})$.

of sonication, whereas the $5 \mathrm{~nm}$ one only presents some disorder in Au NPs packing on the outermost layer.

The cohesion of the multilayer elaborated with small Au NPs thus appears stronger than that prepared with larger ones. This finding is in good agreement with what can be expected from size effects in such nanomaterials. Indeed, the total free volume (tetrahedral and octahedral voids) in a close-packed 2D assembly of monodisperse spherical particles ( 1 or 2 layers for instance) increases with the size of the particles [1,17]. As a result, one expects the cohesion of the ordered assembly of spherical particles to decrease with the increase of the Au NPs diameter. This size-dependent 


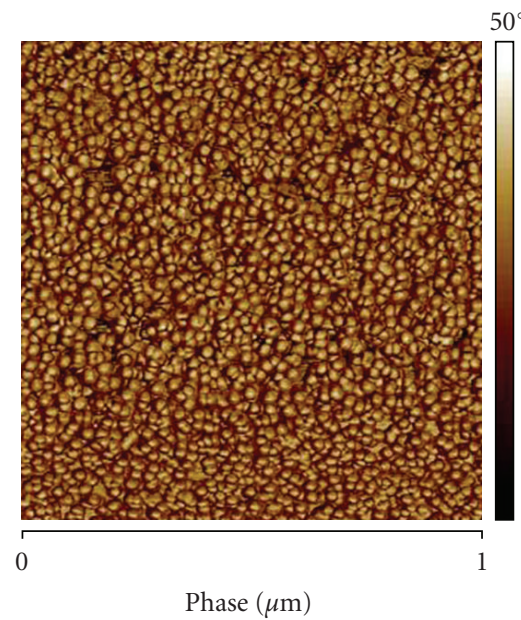

(a)

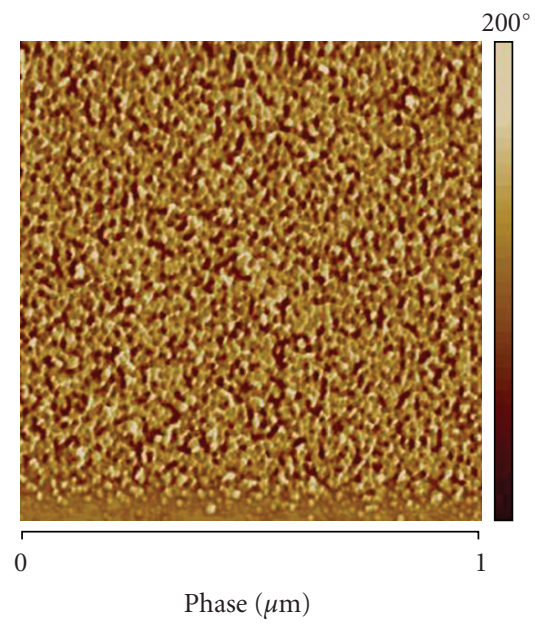

(c)

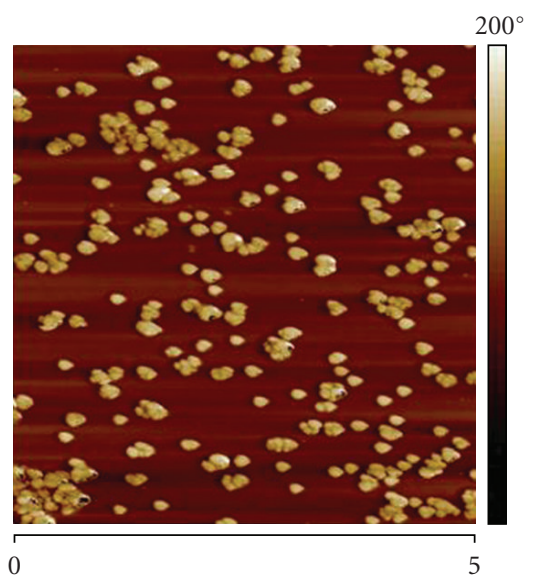

Phase $(\mu \mathrm{m})$

(b)

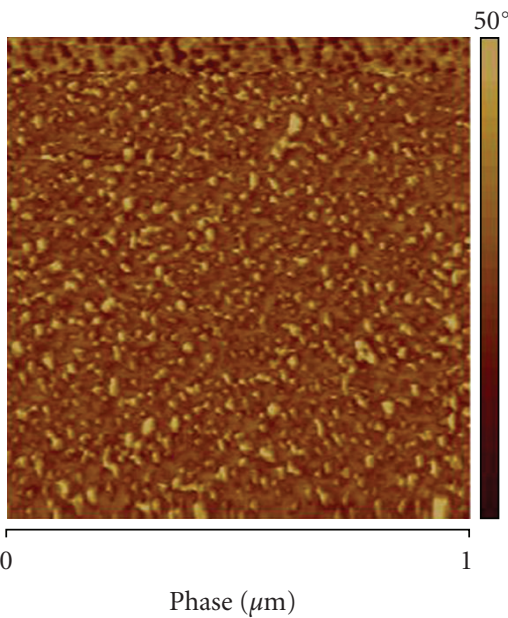

(d)

FIGURE 8: AFM pictures in tapping mode of the fourth layer of the multilayer structure before and after sonication in water for 20 minutes. (a) and (b) correspond to the AFM images of the $15 \mathrm{~nm}$-sized NPs, and (c) and (d), to the $5 \mathrm{~nm}$ Au NPs. $15 \mathrm{~nm}$ Au NPs: (a) before sonication $(1 \times 1 \mu \mathrm{m})$, (b) after sonication $(5 \times 5 \mu \mathrm{m}) ; 5 \mathrm{~nm}$ Au NPs: (c) before sonication $(1 \times 1 \mu \mathrm{m})$, (d) after sonication $(1 \times 1 \mu \mathrm{m})$.

cohesion of NPs-based structures was confirmed in a recent systematic work dealing with the thermomechanical stability of drying structures, built with nanocolloidal $\mathrm{Au}$ particles [18]. These size-dependent properties represent an important result that will have a strong impact on the choice of functional surfaces used either in regenerative or lubrication domain.

The multilayered structures were then used in SERS to characterize the effect of both the layering (number of layers) and the NPs size on their Raman sensitivity.

\subsubsection{Raman Spectrometry on Multilayer Gold Particulate} Film. To promote the above results towards molecular characterization and detection, the SERS spectra of the bare (as-prepared) nanoparticulate multilayers were recorded, compared to standard thermally evaporated gold films, and presented in Figure 9.

The results of Raman spectra show that there is no signal for the thermally evaporated $\mathrm{Au}$ thin film, whose thickness is equivalent to $30 \mathrm{~nm}$. For the nanoparticulate multilayer structure, the Raman analysis shows the emergence of signals, the intensity of which depends on the number of layers.

Basically, for the $15 \mathrm{~nm}$-sized NPs in this experiment, there is no significant band up to two layers (Figure 9(a)). Above 3 layers, the intensity of the main band at $1540 \mathrm{~cm}^{-1}$ appears and increases in intensity as shown in Figure 9(a). The peak observed around $1540 \mathrm{~cm}^{-1}$ corresponds to C$\mathrm{C}$ ring stretching $[14,16]$ of the chain of linker molecules between two layers of particles. The enhancement of Raman intensity between one and five layers is of the order of 50. A small shift and enlargement of the peak is observed with the increase of the number of layers, due to either the coupling between NPs dipoles over a large scale, or the damage of the linker molecules due to the intensity of the laser and the formation of amorphous carbon between the layers, which naturally increases with the number of layers. 


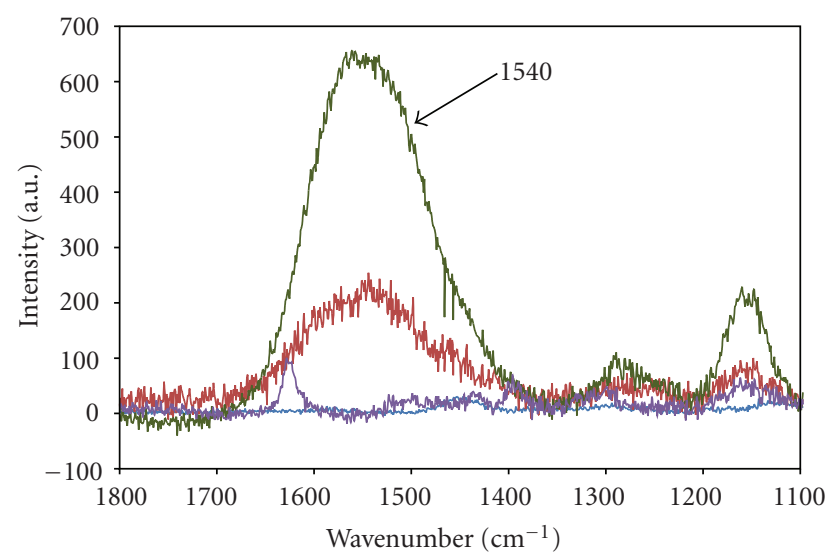

(a)

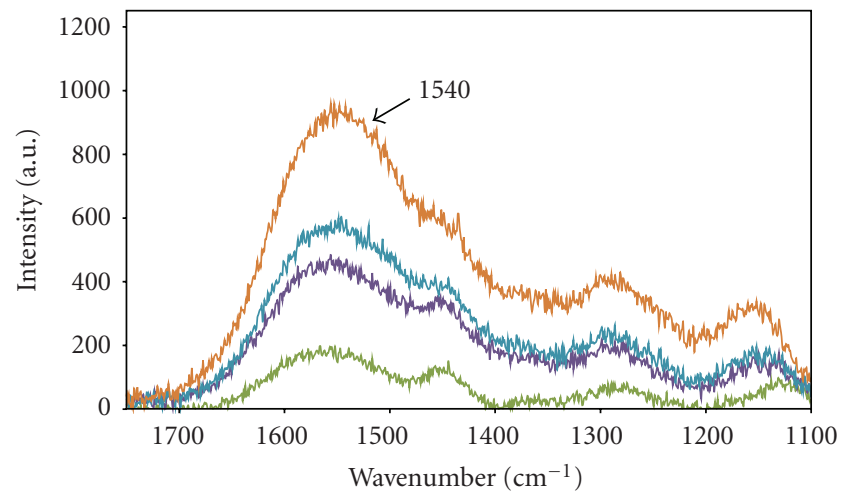

(b)

FIgURE 9: (a) SERS spectra of $15 \mathrm{~nm}$ Au NPs multilayer structure and thermally evaporated Au film. (1) (blue curve) a standard $\mathrm{Au}$ thermally evaporated film of $30 \mathrm{~nm}$, (2) (violet curve) one monolayer of gold nanoparticles deposited onto an SAMs-coated silicon wafer, (3) (red curve) three layers of nanoparticles hybrid architecture, and (4) (dark green curve) five layers of nanoparticles hybrid architecture. (b) SERS spectra of $5 \mathrm{~nm}$ Au NPs multilayer structure (1) (green curve), one monolayer of gold nanoparticles deposited onto an SAMs-coated silicon wafer, (2) (dark blue curve) two layers of nanoparticles hybrid architecture, (3) (blue curve) three layers of nanoparticles hybrid architecture, and (4) (pink curve) four layers of nanoparticles hybrid architecture.

For the $5 \mathrm{~nm}$-sized NPs system, the same main peak was observed. However, contrary to the larger NPs multilayer, the intensity of the main band at $1540 \mathrm{~cm}^{-1}$ was already significant from the first layer, and increases continuously up to the fourth layer. The enhancement of Raman signal between one and four layers is higher compared to the larger NPs multilayer colloidal system, and of the order of 70.

Moreover, by comparing two equivalent multilayer thicknesses made of the two Au NPs sizes (e.g., 3 layers of $5 \mathrm{~nm} \mathrm{Au}$ NPs film versus 1 layer of $15 \mathrm{~nm}$ NPs film), the intensity enhancement factor is of the order of 100. This result definitively shows the powerful enhancement of SERS sensitivity by the sole size effect of NPs entering the surface structure.

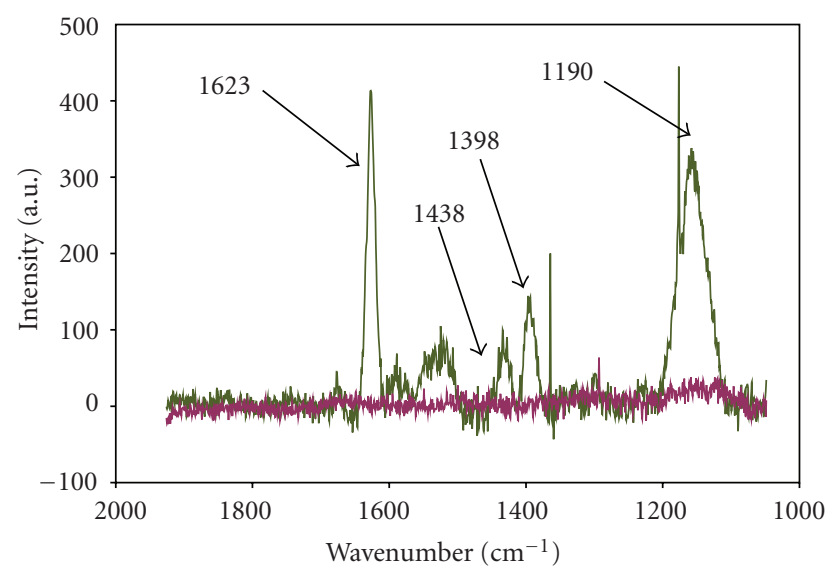

FIGURE 10: SERS spectra of methylene blue adsorbed on a $15 \mathrm{~nm}$ sized NPs multilayer structure and a thermally evaporated Au film; (a) (blue curve) thermally evaporated Au film, (b) (green curve) five layers of gold nanoparticles multilayer architecture.

TABLE 2: Assignment of the main peaks of the SERS spectra of methylene blue adsorbed on NPs multilayer structure.

\begin{tabular}{lc}
\hline $\mathrm{Cm}^{-1}$ & Assignments \\
\hline 1623 & $\mathrm{C}-\mathrm{C}$ and $\mathrm{C}-\mathrm{N}-\mathrm{C}$ \\
1438 & $\mathrm{C}-\mathrm{C}$ \\
1398 & $\mathrm{C}-\mathrm{C}$ \\
& $\mathrm{C}-\mathrm{N}-\mathrm{C}$ \\
1190 & $\mathrm{COO}-$ \\
\hline
\end{tabular}

To test the SERS sensitivity of these NPs size-adjusted multilayers structures, a standard dye molecule, the methylene blue (MB), is used as a probe molecule.

3.2.4. Adsorption Behaviors of Methylene Blue in Gold Nanoparticulate Multilayers Architecture. We used surfaceenhanced Raman spectroscopy (SERS) to detect the adsorption of very small amounts of methylene blue (MB) on the Au NPs multilayer structure, which was compared to the standard, thermally evaporated Au thin film. Prior to SERS measurement, $10^{-7} \mathrm{M}$ of $\mathrm{MB}$ solution was prepared and both the nanoparticulate and thermally evaporated films were immersed for one minute in the diluted solution and then dried in air. A typical SERS spectrum of methylene blue adsorbed on the Au evaporated film and the five layers nanoparticulate structure is shown in Figure 10. The first trivial observation is the weak signal of the $\mathrm{MB}$ on the evaporated Au film, compared to the strong response on the multilayer structures.

The SERS spectra and the characteristic peaks of MB are displayed in Figure 10 and in Table 2.

In the spectra of Figure 10, the main peak at $1623 \mathrm{~cm}^{-1}$ corresponds to the ring stretch modes of $((\nu \mathrm{C}-\mathrm{C})$ and $(\nu \mathrm{C}-$ $\mathrm{N}-\mathrm{C})$ ). At $1438 \mathrm{~cm}^{-1}$, a second weaker peak is assigned to the ring stretch of ( $\nu$ CC). And finally at $1398 \mathrm{~cm}^{-1}$, another medium peak corresponds to both ring stretch modes, $((\nu \mathrm{C}-$ C) and $(\nu \mathrm{C}-\mathrm{N}-\mathrm{C}))$. Our results are in good agreement with 
values previously published $[18,19]$. The strong and medium peak at $1190 \mathrm{~cm}^{-1}$ was previously observed on the SERS spectra of the bare structure as shown in Figure 9. This peak corresponds to the ring stretch $\left(\nu \mathrm{COO}^{-}\right)$of the carboxylate group of the citrate molecules adsorbed onto the Au NPs.

In Figure 11(a), the spectra show the variation of intensity of the main peak of the SERS spectra of MB (at $1623 \mathrm{~cm}^{-1}$ ) as function of the number of layers in the $15 \mathrm{~nm} \mathrm{Au} \mathrm{NPs} \mathrm{multilayer} \mathrm{structure.} \mathrm{At} \mathrm{a} \mathrm{constant}$ NPs surface coverage of each layer, and for a constant $\mathrm{MB}$ concentration, these multilayer structures were shown to create a strong enhancement of the Raman signal of the adsorbed methylene blue (MB). In Figure 11(b), the evolution of the intensity of the main peak at $1623 \mathrm{~cm}^{-1}$ of the MB was plotted versus the number of Au colloidal layers. As the number of layers increases, a regular and sharp increase of the Raman intensity of adsorbed MB is recorded. The linear evolution of the maximum peak intensity versus the number of layers is characteristic of the profile commonly observed in LbL assemblies as described in the literature [14].

These enhanced Raman signals are mainly due to the enhanced electromagnetic field resulting from the coupling over the localized plasmons of the discrete and close-packed $\mathrm{Au}$ NPs in the multilayer structure [1]. By increasing the number of layers, the number of dipoles (the resulting dipole of the multilayer) increases, leading to an amplification of the electromagnetic field of the multilayer. This amplification of the surface Plasmon intensity creates in turn an amplification of the electromagnetic excitation sensed by the molecular species embedded in the multilayer. Therefore, an increase of the SERS intensity can be achieved through an increase of the number of layers, in a surface structure based on a multilayer assembly of Au NPs.

But more interestingly, if we compare the Raman spectrum of an MB molecule adsorbed onto 3 layers of $5 \mathrm{~nm}$ sized $\mathrm{Au}$ NPs to the one adsorbed on a monolayer of $15 \mathrm{~nm}$ sized NPs as displayed in Figure 12, we observe a strong enhancement of the intensity of the Raman signal with a decrease of the NPs size. The amplification of the main peak at $1623 \mathrm{~cm}^{-1}$ corresponding to the $\mathrm{C}-\mathrm{C}$ stretching is particularly well defined. In addition, the SERS enhancement factor is of the order of 100 for equivalent multilayer films thicknesses. This major result particularly emphasizes the size effect of the nanoparticles on SERS properties and well shows the dependence of the optical response on the size of metal binding unit in the nanoparticle assembly. The smaller the particles are, the higher the (surface/volume) ratio corresponding to their $2 \mathrm{D}$ assembly is, and the stronger the optical coupling response is. To complete the experimental results, it should have been interesting to quantify the amount of $\mathrm{MB}$ adsorbed in the multilayer system for each size of NPs. However, no spectroscopic instrumentation was available at the laboratory to determine precisely this value.

Finally, the layer-by-layer technique can be used to create a combined electrostatic-covalent structure of robust Au NPs multilayers. For a given NPs size and shape, the surface coverage of the NPs in a layer, the compactness, and the number of layers in the structure constitute the main

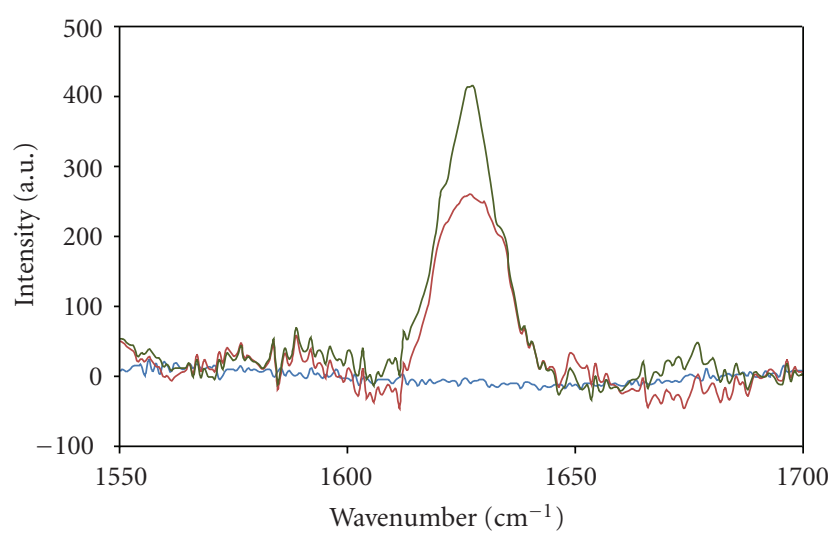

(a)

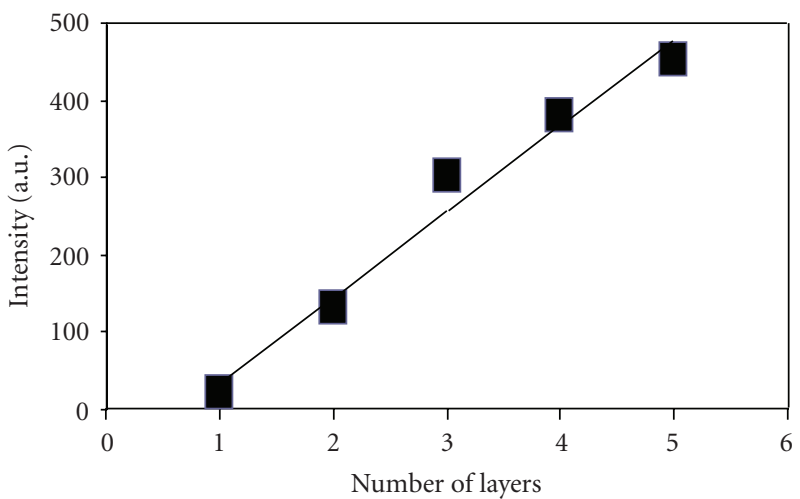

(b)

Figure 11: (a) Zoom in on the main MB peak at $1623 \mathrm{~cm}^{-1}$ of methylene blue adsorbed on a $15 \mathrm{~nm}$ diameter NPs multilayer structure and Au thermal evaporated film obtained by SERS. (i) (blue curve) Au evaporated thermally evaporated film, (ii) (red curve) three layers of nanoparticles hybrid architecture, (iii) (green curve) five layers of gold nanoparticles hybrid architecture. (b) Evolution of the intensity of the main MB peak at $1623 \mathrm{~cm}^{-1}$ of methylene blue adsorbed NPs multilayer structure versus the number of layers.

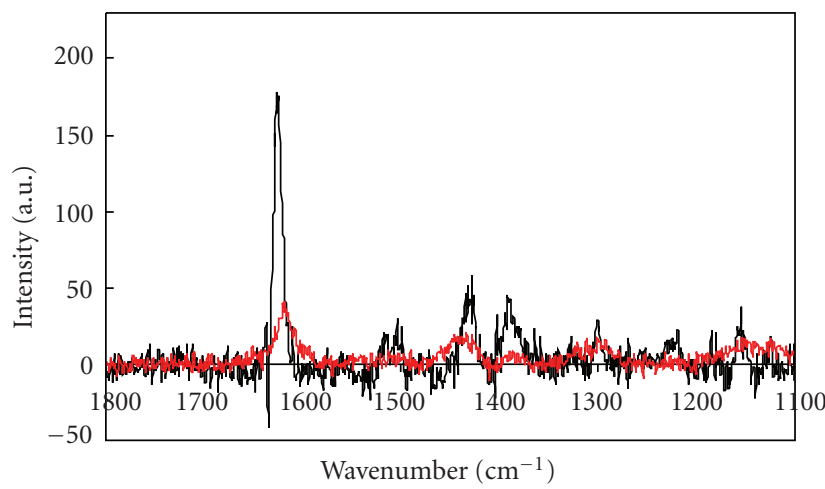

FIGURE 12: SERS spectra of methylene blue adsorbed $15 \mathrm{~nm}$ and $5 \mathrm{~nm}$-sized Au NPs multilayer structure (A). (black curve) 3 layers of $5 \mathrm{~nm}$-sized gold nanoparticles hybrid architecture, (B). (red curve) monolayer of $15 \mathrm{~nm}$-sized gold nanoparticles hybrid architecture. 
parameters, which determine the cohesion and the collective optical properties of the surface structure.

As it has been demonstrated in this paper, the size adjustment of the NPs in multilayered surface structure provides a promising route for elaborating effective SERSactive substrate for the detection of very small amounts of organic molecules in either air or solutions. Moreover, the adjustment of the interlayer distance by the chain length of the linker [4] constitutes an additional tuning parameter of this SERS effect. Hence, the cohesive and adhesive properties, the stability, and the size effect brought by these multilayered architectures will have an important potential of applications in environmental detection, in bioengineery and medicine, or as sensors and optical devices [16].

\section{Conclusion}

In the present paper, a facile fabrication route of a multilayer architecture, alternating organic molecules and gold colloidal nanoparticles onto silicon wafer, had been successfully developed. The robustness of the superlattice structure has been demonstrated by the strong combined electrostatic and covalent binding between molecules and nanoparticles. These interactions enable to insure the compactness of the superstructure and a high surface coverage of each individual layer. Finally, these multilayer structures of $\mathrm{Au}$ nanoparticles have proved to be an effective analytical tool when used to detect very low amount of methylene blue dye adsorbed on the structures, by surface-enhanced Raman spectroscopy (SERS). Furthermore, we showed that both the cohesion and the SERS sensitivity of the multilayer structures were significantly enhanced by decreasing the size of the $\mathrm{Au}$ nanoparticles building units, bringing a clear evidence of size effects in nanostructured materials. The combination of this proved size effect with the shape and the organization of nanocolloidal metal particles in general, and of Au nanocolloids in particular, provides a new and effective tuning parameter towards the improvement of the stability and sensitivity of nanostructure-based devices.

\section{Supporting Information}

A short description of the different characterization tools is provided in this section.

Ellipsometry. Film thicknesses, including the grafted amineterminated monolayer and the Au NPs layers, were determined by null ellipsometry (Multiskop, Optrel, Berlin, Germany). All measurements were done under ambient conditions at an incidence angle of $70^{\circ}$, using an Nd-YAG laser $(\lambda=532 \mathrm{~nm})$. The film thickness was calculated using the Elli software (Optrel).

Atomic Force Microscopy. Surface images were acquired with an atomic force microscope in Tapping mode powered by a Nanoscope IV controller (Digital Instruments, Santa Barbara, CA) using Silicon AFM tips (Nanosensor, Park Scientific) with a spring constant of $k=20 \mathrm{~N} \mathrm{~m}^{-1}$.
UV-Vis and Raman Instrumentation. The UV-Vis spectra of the gold solutions were generated using a Perkin-Elmer Lambda 35 UV-Vis spectrophotometer. The Raman spectra were obtained with a Horiba Labram Raman equipment, using an Olympus BX40 microscope and an excitation source of $632.81 \mathrm{~nm}$. The laser power at the sample was $5 \mathrm{~mW}$, and the resolution was set at $1.6 \mathrm{~cm}^{-1}$.

Transmission Electron Microscopy. The size and morphology of nanoparticles were analyzed by transmission electron microscopy (TEM) performed on a Philips CM 200 at an accelerating voltage of $200 \mathrm{kV}$. TEM samples were prepared by dipping 400 mesh carbon-coated copper grid into the colloidal suspension of nanoparticles and air dried for $24 \mathrm{~h}$ prior to analysis.

\section{Acknowledgment}

This work was supported by the Centre National de la Recherche Scientifique (CNRS).

\section{References}

[1] J. P. Camden, J. A. Dieringer, J. Zhao, and R. P. Van Duyne, "Controlled plasmonic nanostructures for surface-enhanced spectroscopy and sensing," Accounts of Chemical Research, vol. 41, no. 12, pp. 1653-1661, 2008.

[2] J. Tien, A. Terfort, and G. M. Whitesides, "Microfabrication through electrostatic self-assembly," Langmuir, vol. 13, no. 20, pp. 5349-5355, 1997.

[3] T. Kraus, L. Malaquin, H. Schmid, W. Riess, N. D. Spencer, and $\mathrm{H}$. Wolf, "Nanoparticle printing with single-particle resolution," Nature Nanotechnology, vol. 2, no. 9, pp. 570-576, 2007.

[4] P. J. G. Goulet, D. S. dos Santos Jr., R. A. Alvarez-Puebla, O. N. Oliveira, and R. F. Aroca, "Surface-enhanced raman scattering on dendrimer/metallic nanoparticle layer-by-layer film substrates," Langmuir, vol. 21, no. 12, pp. 5576-5581, 2005.

[5] X. Li, W. Xu, J. Zhang et al., "Self-assembled metal colloid films: two approaches for preparing new SERS active substrates," Langmuir, vol. 20, no. 4, pp. 1298-1304, 2004.

[6] B. R. Cuenya, S. H. Baeck, T. F. Jaramillo, and E. W. McFarland, "Size- and support-dependent electronic and catalytic properties of Au /Au nanoparticles synthesized from Block Copolymer Micelles," Journal of the American Chemical Society, vol. 125, no. 42, pp. 12928-12934, 2003.

[7] M. B. Cortie and E. van der Lingen, "Catalytic gold nanoparticles," Materials Forum, vol. 26, pp. 1-14, 2002.

[8] M. C. Daniel and D. Astruc, "Gold nanoparticles: assembly, supramolecular chemistry, quantum-size-related properties, and applications toward biology, catalysis, and nanotechnology," Chemical Reviews, vol. 104, no. 1, pp. 293-346, 2004.

[9] R. K. Iler, "Multilayers of colloidal particles," Journal of Colloid and Interface Science, vol. 21, no. 6, pp. 569-594, 1966.

[10] E. Leguen, A. Chassepot, G. Decher, P. Schaaf, J. C. Voegel, and N. Jessel, "Bioactive coatings based on polyelectrolyte multilayer architectures functionalized by embedded proteins, peptides or drugs," Biomolecular Engineering, vol. 24, no. 1, pp. 33-41, 2007. 
[11] X. Hu, W. Cheng, T. Wang, Y. Wang, E. Wang, and S. Dong, "Fabrication, characterization, and application in SERS of selfassembled polyelectrolyte—gold nanorod multilayered films," Journal of Physical Chemistry B, vol. 109, no. 41, pp. 1938519389, 2005.

[12] G. Frens, "Controlled nucletion for the regulation of the particle size in monodiperse gold suspensions," Nature, vol. 241, pp. 20-22, 1973.

[13] M. Dragoman and D. Dragoman, "Plasmonics: Applications to nanoscale terahertz and optical devices," Progress in Quantum Electronics, vol. 32, no. 1, pp. 1-41, 2008.

[14] K. C. Grabar, K. J. Allison, B. E. Baker et al., "Two-dimensional arrays of colloidal gold particles: a flexible approach to macroscopic metal surfaces," Langmuir, vol. 12, no. 10, pp. 2353-2361, 1996.

[15] P. B. Johnson and R. W. Christy, "Optical constants of the noble metals," Physical Review B, vol. 6, no. 12, pp. 4370-4379, 1972.

[16] T. Somanathan and A. Pandurangan, "Direct synthesis of vanadium substituted mesoporous MCM-41 molecular sieves: a systematic study for the growth of SWNTs," Journal of Porous Materials, vol. 16, no. 6, pp. 657-665, 2009.

[17] B. R. Cuenya, S. H. Baeck, T. F. Jaramillo, and E. W. McFarland, "Size- and support-dependent electronic and catalytic properties of $\mathrm{Au} / \mathrm{Au}$ nanoparticles synthesized from block copolymer micelles," Journal of the American Chemical Society, vol. 125, no. 42, pp. 12928-12934, 2003.

[18] S. Darwich, K. Mougin, and H. Haidara, "Complex aggregation patterns in drying nanocolloidal suspensions: size matters when it comes to the thermomechanical stability of nanoparticle-based structures," Langmuir, vol. 26, no. 22, pp. 16928-16933, 2010.

[19] G. Laurent, N. Félidj, J. Grand et al., "Raman scattering images and spectra of gold ring arrays," Physical Review B, vol. 73, no. 24, Article ID 245417, 2006. 


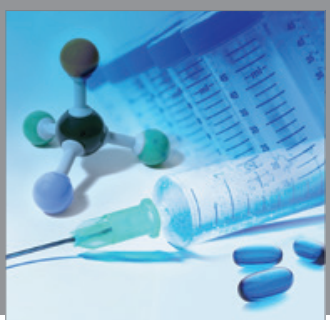

International Journal of

Medicinal Chemistry

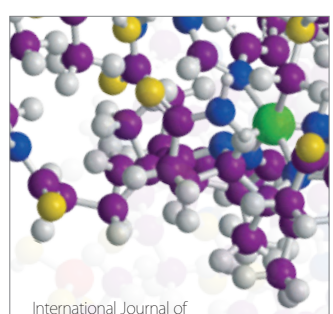

Carbohydrate Chemistry

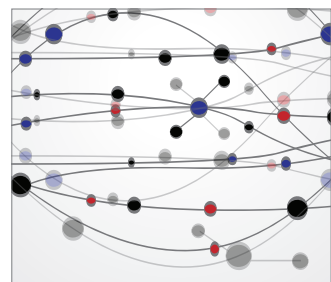

The Scientific World Journal
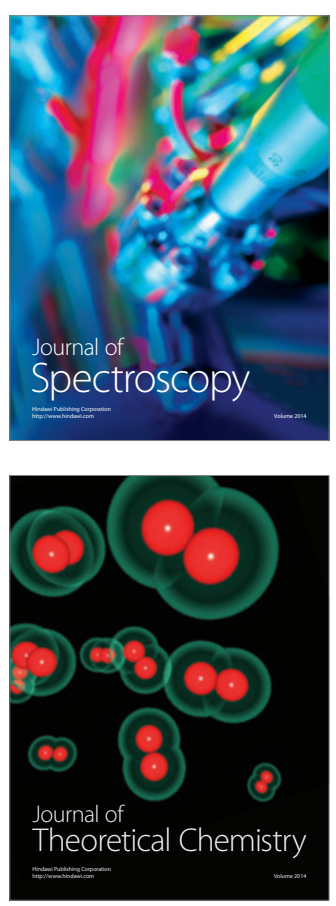
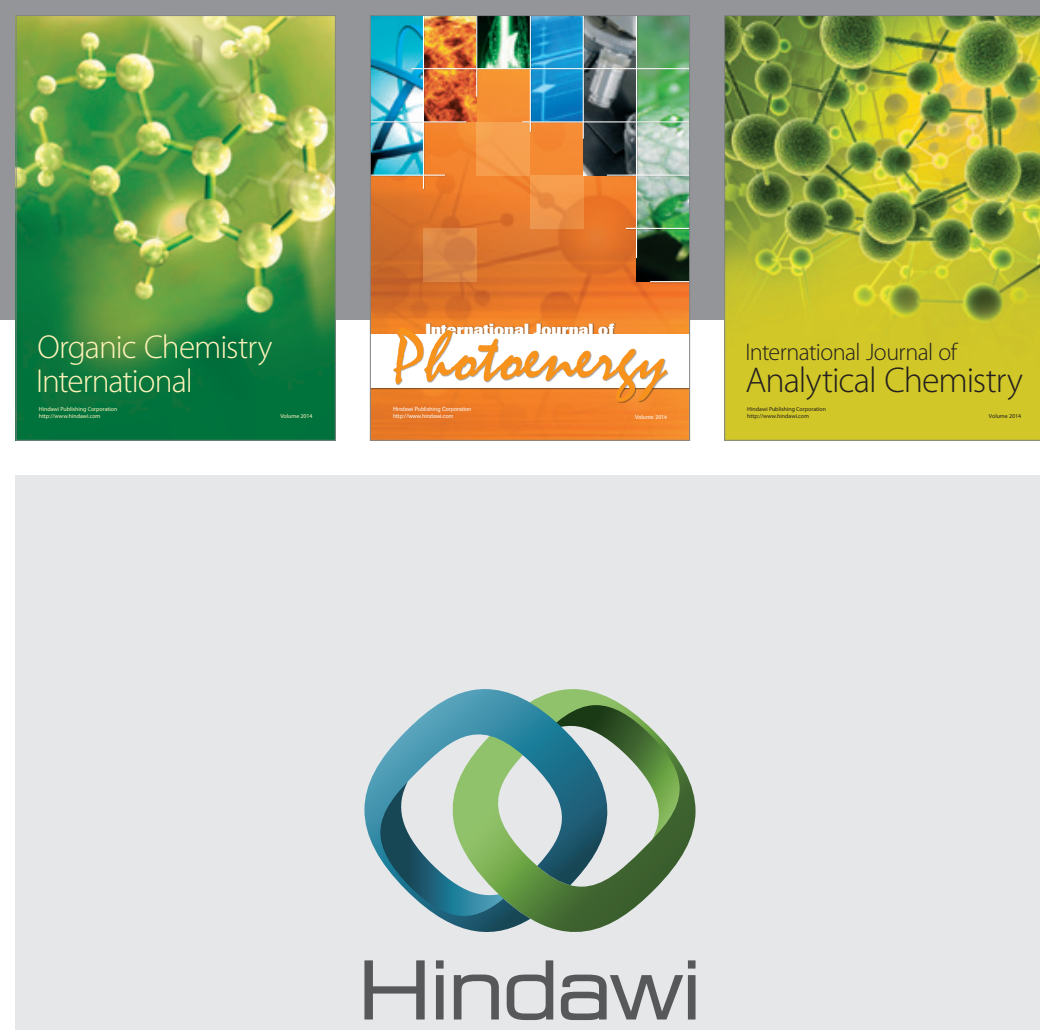

Submit your manuscripts at

http://www.hindawi.com
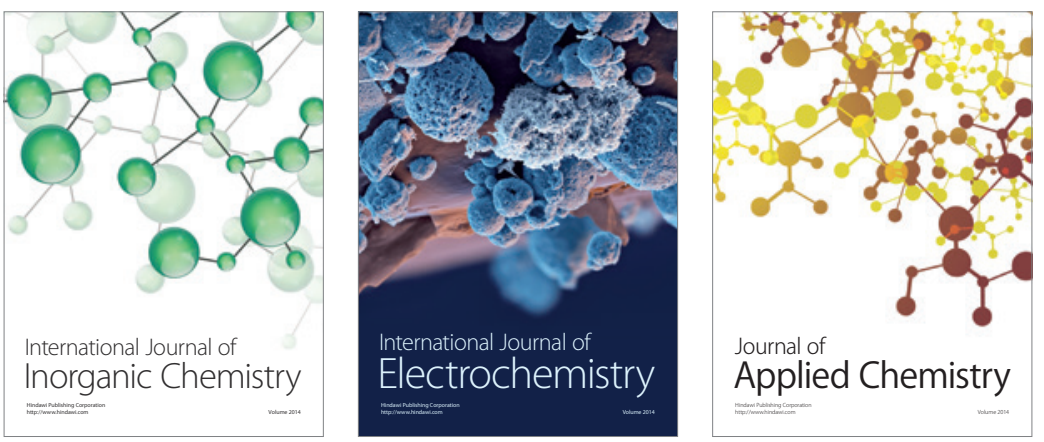

Journal of

Applied Chemistry
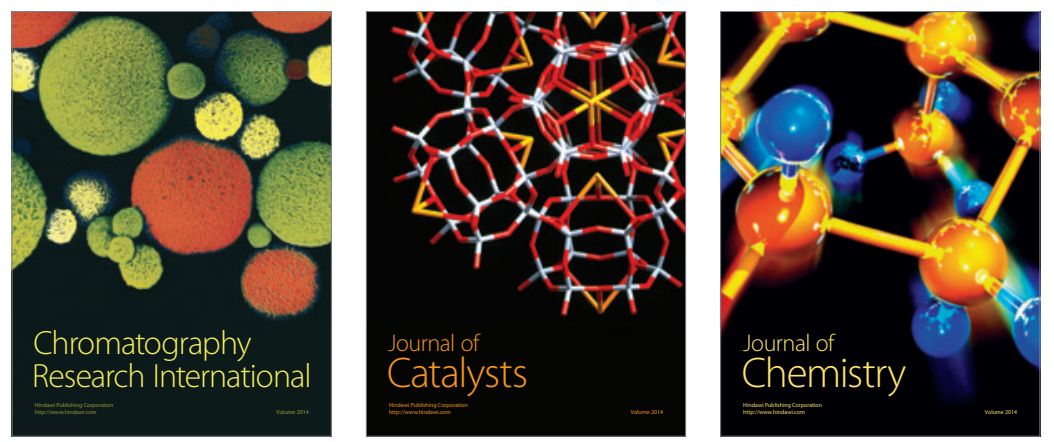
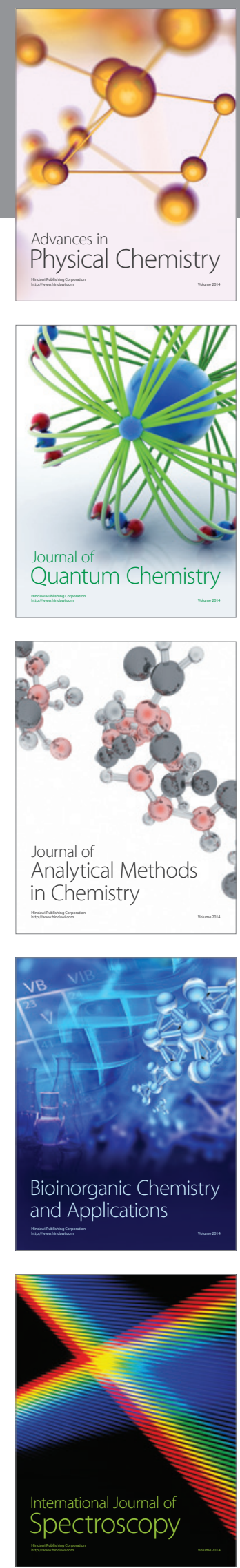